\title{
USE OF AN AMPLATZER DEVICE FOR ENDOBRONCHIAL CLOSURE OF BRONCHOPLERURAL FISTULA FOLLOWING A RIGHT PNEUMONECTOMY
}

Galve Marqués, AII , Portas Gonzalez, M1, Chamorro García, E¹, Alonso Mateo, $\mathrm{M}^{1}$, Diez-delhoyo, $\mathrm{F}^{2}$, Melone Fiorito, $\mathrm{A}^{1}$

${ }^{1}$ Servicio de Anestesiologia y Reanimacion, Hospitat General Universitario Gregorio Marañon, Madrid. España 2Servicio de Cardiologia, Hospital General Universitario Gregorio Marañon, Madrid. España

\section{Background}

Bronchopleural fistula (BPF) is a dreaded and severe complication of pneumonectomy because of its high morbidity and mortality rates. Its incidence ranges between 3 and $26 \%$ approximately. There are different surgical and endoscopic therapeutic options to solve the BPF. We present a case of endobronchial closure of a BPF using an Amplatzer septal occluder (ASO), a device originally designed for transcatheter closure of cardiac septal defects.

\section{Case Report}

A 67-year-old man was admitted into our ICU 7 days after right pneumonectomy for adenocarcinoma was performed. He developed respiratory insufficiency needing Optiflow $45 \mathrm{lpm}$ and $\mathrm{FiO}_{2}$ 0,45. Bronchoscopy revealed a large BPF (7 mm size) in the medial edge of the right pneumonectomy stump. Treatment with antibiotics, cures, chest tube drainage and thoracostomy failed. Thoracic surgeons dismissed surgical treatment. Interventional pneumologists and cardiologists decided to place a $10 \mathrm{~mm}$ ASO through the BPF. The procedure was successfully performed under mild sedation with complete closure of the fistula. The patient was transferred to the ward 20 days after the endoscopic procedure. Intubation was not needed during the procedure, only Optiflow was used with $\mathrm{FiO}_{2}$ up to 0.5. A few months later, TRAM flap was performed, this procedure was delayed because of a pulmonary embolism during the recovery period.

\section{Discussion}

Traditional surgical therapies for BPF have included primary repair or delayed closure with creation of a cavity with or without omental or muscle flap reinforcement. Newer less invasive techniques utilize bronchoscopy to deliver bioglues, stents or coils and constitute another suitable treatment option. The ASO is the standard of care for transcatheter atrial septal defect closure. It is a self-expandable, braided ninitol and interwoven polyester double-disk device. In our case, a guide wire was introduced through the fistula under direct bronchoscopic visualization. The Amplatzer device was then placed by pulling gradually. First of all, the distal disk was pulled back against the distal side of the defect. The central waist filled the defect and after that the second disk was placed on the proximal side of the defect.

\section{Learning Point}

The ASO can be useful tool for the endoscopic closure of BPF following pneumonectomy for patients in poor general condition without a surgical treatment option. It is a non invasive technique well tolerated under mild sedation to keep in mind.

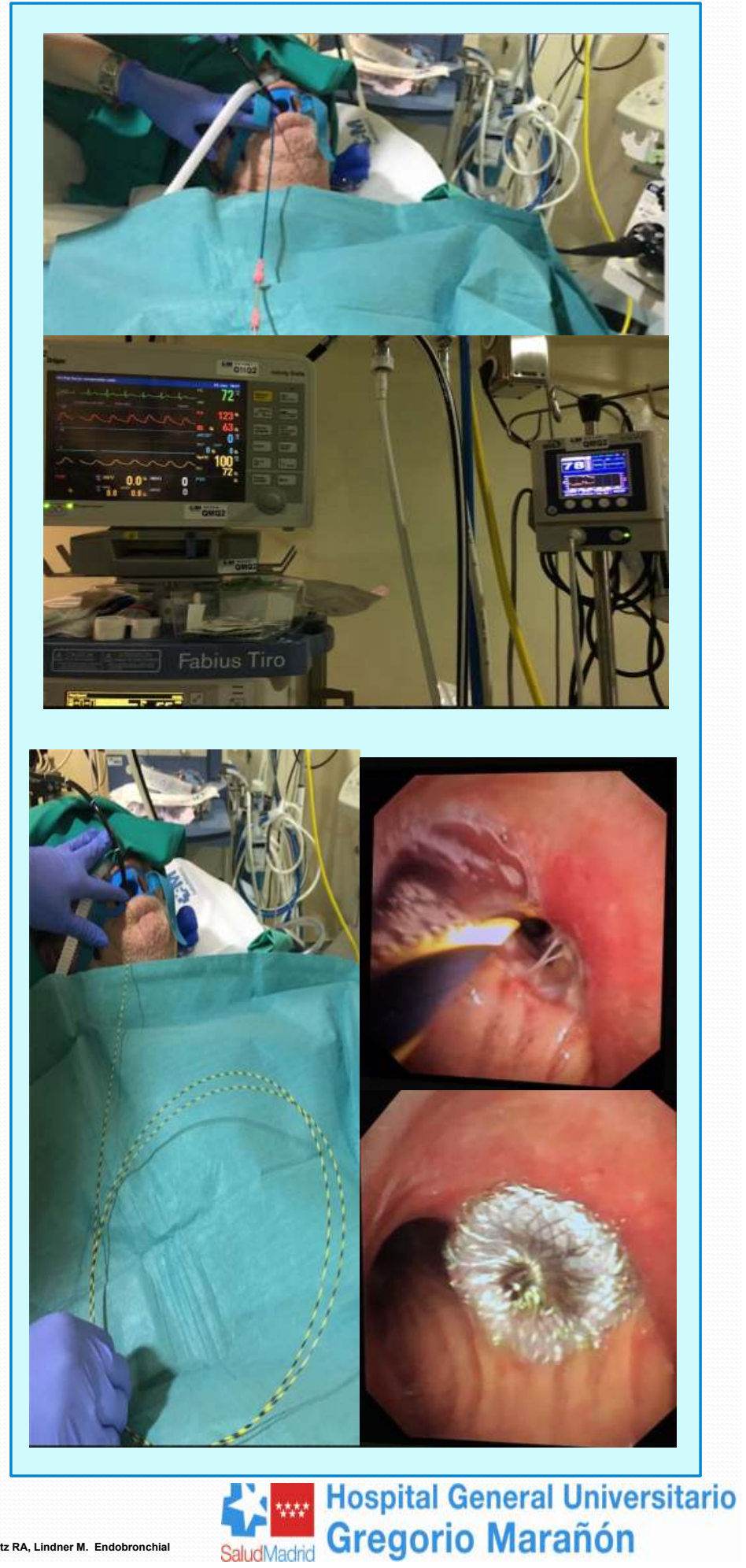

